\title{
COLLOID SCIENCE AND SOME OF ITS INDUSTRIAL APPLICATIONS*
}

\author{
By Dr. R. MATALON \\ Department of Physical Chemistry, King's College, London
}

\begin{abstract}
T $T$ is undoubtedly true that fundamental research carried out in surface chemistry for more than forty years has now enabled technicians and engineers to find new and simpler methods for solving industrial problems as well as improving current processes in practically all fields : for example, textiles, metallurgy, cosmetics, drugs, paints and lubricants. In view of the importance of this growing field of science, some of the latest theoretical developments will be discussed with the object of encouraging applied research, using as a basis the fundamental approach that is now the subject of inquiry in the universities, and which, it may be hoped, will be taken up by other institutions conducting research in pure and applied science.
\end{abstract}

Considering first in its broadest sense orientated adsorption and its importance in determining colloidal behaviour, in which the pioneering work was done by Sir William Hardy, Lord Rayleigh and Devaux, the main factors are gaseous, liquid expanded, liquid condensed, viscous elastic and solid surface-films in relation to the molecular structure of the spread compound, to molecular packing, and to the cohesive forces such as are brought about by van der Waals forces, polar forces, hydrogen bonding and the like; the main observations on surface-films have been made with the Langmuir balance. The more recent approach is that of the school of Sir Eric Rideal on the study of reactions in surface-films : the extent to which the rate of reactions is affected by orientation and packing has been demonstrated by A. Hughes and Rideal for the oxidation of fatty acids, by A. E. Alexander for the hydrolysis of esters, and by J. H. Schulman and Hughes for the Iypolysis by enzymes. In addition, molecular interaction in monolayers has been studied extensively by Rideal, Schulman, E. Stenhagen, E. G. Cockbain and R. Matalon.

The surface-film technique has indeed enabled the study of the reaction of tanning of proteins using tannic acid and phenols as well as polyvalent metallic ions such as ferric chloride. On the other hand, when the molecular interaction results in the 'penetration' of the solute molecules into the surface-film, a mixed structure of both components is observed. This type of interaction produces a solution complex or a stoichiometric association depending on the relative affinity of the interacting molecules. Penetration is observed at both the air/water and the oil/water interfaces. In the latter case, the relation between the surface pressures of the mixed film $\pi_{m}$ and that of the single components $\pi_{1}$ and $\pi_{2}$, present alone at the same interface, may be exemplified for the system cholesterol - laurylsulphate. Whereas, as a general rule, the more surface-active component displaces from the surface the less active one, when penetration occurs the final surface pressure of the system exceeds that of any one of the single components $\pi_{1}$ and $\pi_{2}$, and in dilute systems $\pi_{m}=\pi_{1}+\pi_{2}$. When the inter-

* Substance of five lectures delivered at King's College, London, on * Substance of five lectures delivered at King's College,
January 13 and 27, February 10 and 24 , and March 10. acted mixed film is liquid, molecular interaction in bulk solution, as shown by Dervichian, results in the appearance of myelinic figures and tactoids.

There are a number of examples of the role of penetrated films in spontaneous emulsification. Thus the presence of a trace of a penetrating substance such as iso-octylalcohol in an alkylsulphonate greatly enhances the wetting of fibres and paper. The effect of adsorption on solids is illustrated by a simple experiment: copper chromate precipitated by the reaction of copper sulphate and potassium chromate is produced in the flocculated state when a trace of sodium oleate is added to the chromate. Finally, the periodicity observed in the Liesegang phenomenon is exemplified in the case of silver chromate, in which the adsorption complex gelatin-silver chromate is responsible for the periodicity observed.

Turning now to foams and the fundamental properties connected with foaming, the first requirement is that the molecules must rapidly diffuse from the bulk to the surface, the second that the adsorbed molecules must lead to a surface layer which is either condensed viscous or elastic, and the third that the surface layer must be somehow insolubilized-that is, showing a slow rate of desorption.

The traction apparatus developed by me shows the behaviour of surfaces on traction and retraction with saponins, proteins, beer and the synthetic surface-active agents. Saponins and proteins lead to an irreversible pattern on traction and retraction, whereas the alkylsulphate shows a reversible behaviour. Foaming may be done with anionic and cationic derivatives and also with non-ionics, as exemplified by the good foaming obtained with a condensate of heptylalcohol and six molecules of ethylene oxide. The part played by additives such as methylcellulose, alginic acid and polyvinyl alcohol in stabilizing the foam can be attributed to the more coherent structure obtained as a result of interaction with the foaming agent. There is a grave lack of quantitative work on the equally important field of defoaming. One of the important factors in defoaming is the displacement of a coherent surface layer by a compound, leading to a higher surface pressure and to a gaseous type of film. Defoaming of fermentation baths by the addition of alkylbenzenesulphonate is a direct example of this.

The useful applications of foaming in industry are manifold, as is indicated by the following selection : in fire-fighting; in food, such as in the manufacture of aerated chocolates, creams and meringues; in cosmetics, shampoos and washing powders; in building materials, such as porous concrete and light aggregates; and in the rubber industry, where porous rubber of both types may be obtained, those with isolated cells and with intercommunicating cells. On the other hand, the various instances where foaming is undesirable and combated are in the manufacture of photographic paper; in the course of distillation; in the working of lubricating oils; during fermentation; and in drilling muds. 
Another aspect of colloids is that of emulsions, and the factors of importance in this colloidal state are : the emulsifier and its orientation at the interface; the mechanical properties of the interfacial film; its electrical properties; the relative density of the phases in contact; and the volume phase ratio. The role of the hydrophobic-hydrophilic balance of the emulsifier in determining the type of emulsion obtained is shown in the case of the ethylene oxide condensate with long-chain polar compounds. The nature of the oil must also be considered in relation to the emulsifier, and it can be shown that oleyl alcohol condensed with three ethylene oxide molecules leads to very good oil-in-water emulsions of spindle oil. For triolein, optimum emulsification is obtained with eight to ten molecules of ethylene oxide condensed on the oleyl alcohol. For oleic acid the emulsification is satisfactory with a condensate of fifteen molecules of ethylene oxide on the oleyl alcohol.

In considering the stability of the emulsion in relation to creaming and coalescence, the role of thickeners such as gums, clays, polyvinylalcohols in stabilizing emulsions is attributed to the effect of these materials in slowing down the creaming. Coalescence can now be studied directly by the traction curve of laminæ and by the life-time of droplets at the oil-water interface; the latter method bas recently been developed by Cockbain and Roberts. In general, need for further study of emulsions lies in the importance of their role in industry, as, for example, in food for the manufacture of margarine, ice cream, mayonnaise, etc.; in textiles for the lubrication of yarn, the fulling of wool, sizing, mothproofing, etc.; the printing of cotton; the processing of leather; paints and distempers; polishes; insecticidal and fungicidal preparations; cutting-oils to aid the machining of metal, leading to higher cutting speeds and better finish of the surface; and the water-oil emulsions used for drilling muds.

Turning next to the phenomenon of wetting in colloids, the principles of wetting solids, the methods used for describing and studying this interesting property and finally the various applications of wettability in industrial practice must be considered. The measurement of the contact angle $\theta$ at the solid/liquid/air interface may be made by various techniques such as the capillary rise, the tilting plate and by direct observation using a microscope or any other magnifying equipment. The recent approach of J. Guastalla and his co-workers on the measurement of the adhesion tension is simple and entirely satisfactory for the study of wetting. The traction and retraction curve obtained with a solid plate makes it possible to determine the advancing and receding contact angle by direct applications of the formula $F=\gamma \cos \theta$ on a liquid surface of tension $\gamma$.

In the problem of dispersing and suspending solids, the important factors are particle size and adsorption at the interface of the added wetting agents and builders. Wetting agents find numerous and important applications in industry. Thus, in the pickling of metals, where the introduction of a wetting agent reduces the consumption of acid, loss of metal and processing time, a brighter surface is obtained. In electroplating, wetting agents prevent the adhesion of hydrogen bubbles, as well as control the structure of the deposit (for example, in bright nickel plating), and in the cleaning of met al they remove grease and dirt from surfaces. In soldering, a suitable wetting agent will enable spreading of the zinc chloride-ammonium chloride fluxes, and in the phosphating of steel 0.02 per cent dodecylbenzene sulphonate produces a uniformly treated layer. Phenolic bodies and short-chain alkylsulphonates ensure rapid mercerization of cotton, and wetting agents are good levelling agents in the dyeing of textiles; non-crystalline wetting agents, liquids or pastes, effect quick re-wetting of the textiles after drying. Other uses of wetting agents are in the extinguishing of fires, in accelerating reactions, such as the development of photographic films, and in the ethylation of cellulose, etc. Wetting and dispersion of solids play an important part in the manufacture of paints and varnishes, metallic soaps being here used for promoting wetting and quick dispersion of the pigments in the vehicle. Alkylbenzene and alkylnaphthalene are added in lubricating oils for the dispersion of carbon black. Finally, wetting agents play an important part in the processing of wettable powders used in medicine, in insecticides and in fungicides.

Dealing now with precipitation and growth regulation in supersaturated solutions and in precipitation reactions, a factor of importance is the metastability of the supersaturated system, which may be considered in parallel with the effect of mechanical shock, dust impurities or nuclei. The effect of interfacial forces in determining crystallization is exemplified in the overgrowth phenomenon, and excellent photographs obtained by Dr. M. Blackman and Mr. N. D. Liesegarten (Imperial College of Science and Technology, London) demonstrate the results obtained with potassium chloride, ammonium iodide, rubidium iodide and potassium bromide on mica.

Apart from the effect of the solvent in determining crystal habit, various additives, anionic or cationic, also play a part, as may be demonstrated by the effect of these surface-active agents on the crystal habit of succinic acid. Inhibitors of crystallization have been studied in the case of copper sulphate by A. Packter and myself, and our observations show that as a result of adsorption the time necessary for the appearance of the first visible crystals is increased, and that with increasing addition of inhibitor the crystal size first increases and then decreases again. The effect of sodium laurylsulphate in stratifying copper sulphate crystals is attributed to the adsorption on the copper ion of the long hydrocarbon chain, and the stratification of lead nitrate by agar is paralleled with the rhythmicity of the lead salts in agar.

The conditions affecting precipitation reactions are the primary factors of molecular ratio, concentration and temperature, and secondary factors of $p H$ and other added reagents. The high degree of supersaturation observed in precipitation reactions is ascribed to precipitation complexes, such as $\mathrm{AgCl} \mathrm{Ag}^{+}$, $\mathrm{Ag}_{2} \mathrm{CrO}_{4} \mathrm{Ag}^{+}$, etc. Precipitation inhibitors act by adsorption on to the precipitated sol and thus prevent crystal growth. Finally, the Liesegang phenomenon is a special case of precipitation reaction where adsorption on the precipitate is very pronounced. In general, the importance of controlling precipitation and growth of crystals is very great in the various applications of colloids, such as the preparation of stable sols in medicine, in the photographic industry, in electroplating, etc., and in the production of large crystals. 